\title{
Spin-Rotation Interaction and Anisotropic Chemical Shift in ${ }^{13} \mathrm{CS}_{2}{ }^{*}$
}

\author{
H. W. Spiess, D. Schweitzer, U. Haeberlen, and K. H. Hausser \\ Max Planck Institut, Abteilung Molekulare Physik, Heidelberg, Jahnstrasse 29
}

Received February 1, 1971; accepted, March 23, 1971

\begin{abstract}
The ${ }^{13} \mathrm{C}$ nuclear spin-lattice relaxation time $T_{1}$ was studied in liquid $\mathrm{CS}_{2}$ from $-106^{\circ} \mathrm{C}$ to $+35^{\circ} \mathrm{C}$ at resonance frequencies of 14,30 , and $62 \mathrm{MHz}$. The relaxation is caused by anisotropic chemical shift and spin-rotation interaction. It is shown that for linear molecules the spin-rotation constant $C$ and the anisotropy of the chemical shift $\Delta \sigma$ can be obtained from the relaxation rates without use of adjustable parameters. The analysis yields: $C=-13.8 \pm 1.4 \mathrm{kHz}$ and $\Delta \sigma=438 \pm 44 \mathrm{ppm}$.
\end{abstract}

\section{INTRODUCTION}

Several mechanisms are known to contribute to the spin-lattice relaxation time $T_{1}$ of a nucleus with spin $I=1 / 2$ in pure diamagnetic liquids, e.g., dipolar coupling, anisotropic chemical shift, and spin-rotation interaction. In most of the systems studied so far, the relaxation rates are dominated by the dipolar coupling. For the ${ }^{13} \mathrm{C}$ nucleus in $\mathrm{CS}_{2}$, on the other hand, the latter two mechanisms are expected to determine $T_{1}$. The possibility that anisotropic chemical shift might be a significant relaxation mechanism in $\mathrm{CS}_{2}$ was first pointed out by McConnell and Holm (1). As already mentioned by Abragam (2), "the obvious check of changing the resonance frequency $\omega$ and investigating whether $1 / T_{1} \propto \omega^{2}$ does not seem to have been made." By use of a superconducting magnet, it is now possible to study $T_{1}$ at higher frequencies, and data are presented below taken at 14, 30, and $62 \mathrm{MHz}$. Spin-rotation interaction as a relaxation mechanism for $\mathrm{CS}_{2}$ has been mentioned recently by Lippmaa et al. (3). In a theoretical study of the contributions to $T_{1}$ of spin-rotation interaction and anisotropic chemical shift, Deutch and Waugh (4) concluded that both mechanisms would contribute appreciably to $T_{1}$ for ${ }^{13} \mathrm{C}$ in simple molecules like $\mathrm{CO}, \mathrm{CO}_{2}$, and $\mathrm{CS}_{2}$.

The spin-rotation interaction is closely related to the paramagnetic part of the chemical shift $(5,6)$. For lincar molecules, this relation may be extended to yield the anisotropy of the chemical shift $\Delta \sigma$ as a function of the spin-rotation constant $C$ to a good approximation. By multiplication of the individual relaxation rates, due to spin-rotation interaction and anisotropic chemical shift, the correlation times for both interactions can be eliminated (4) and a second equation relating $\Delta \sigma$ and $C$

* Presented at the "12th Experimental NMR Conference", February 1971, Gainesville, Florida, USA. 
is obtained. Thus both quantities can be determined from relaxation studies without use of adjustable parameters. In order to get the necessary information, $T_{1}$ has to be studied as a function of $\omega$ and the temperature $T$.

\section{CHEMICAL SHIFT AND SPIN-ROTATION INTERACTION IN LINEAR MOLECULES}

The chemical shift tensor $\hat{\sigma}$ is usually represented as the sum of a diamagnetic part $\hat{\sigma}^{d}$ and a paramagnetic part $\hat{\sigma}^{p}$ :

$$
\hat{\sigma}=\hat{\sigma}^{d}+\hat{\sigma}^{p} \text {. }
$$

Taking the $C_{\infty}$ axis of the diamagnetic linear molecule as the $z$ axis we note that $\sigma_{z z}^{p}=\sigma_{\|}^{p}$ vanishes, because the angular momentum operator $L_{z}$ commutes with the total electronic Hamiltonian (2, p. 182). For $\sigma_{x x}^{p}=\sigma_{y y}^{p}=\sigma_{\perp}^{p}$, we have $(5,6)$

$$
\sigma_{\perp}^{p}=\frac{e^{2}}{2 m c^{2}}\left(\frac{\Theta c}{e \mu_{0} g_{K}} C_{K}-\sum_{\alpha}^{\prime} \frac{N_{\alpha}}{r_{\alpha}}\right)
$$

where $C_{K}=C_{K \perp}$ is the spin-rotation constant ${ }^{1}$ of the $K$-nucleus. Note that, for a linear molecule, $C_{K \|}$ vanishes. $\Theta$ is the moment of inertia of the molecule, $\mu_{0}, g_{K}$ are the nuclear magneton and the nuclear $g$-factor of the $K$-nucleus, respectively, $N_{\alpha}$ is the atomic number of the $\alpha$-nucleus, and $r_{\alpha}$ the internuclear distance between $\alpha$ - and $K$-nuclei. The prime on the summation indicates $\alpha \neq K$. For the diamagnetic shift, we use the approximate formula, derived by Flygare and Goodisman (7),

$$
\sigma_{a v}^{d}=\sigma_{a v}^{d}(\text { free atom })+\frac{e^{2}}{3 m c^{2}} \sum_{\alpha}^{\prime} \frac{N_{\alpha}}{r_{\alpha}}
$$

valid for the isotropic part. These authors also show (7) that similar equations hold for the individual components of $\hat{\sigma}^{d}$ as well to a good approximation.

$$
\sigma_{i i}^{d}=\sigma_{\mathrm{av}}^{d}(\text { free atom })+\frac{e^{2}}{2 m c^{2}} \sum_{\alpha}^{\prime} \frac{N_{\alpha}}{r_{\alpha}^{3}}\left(j_{\alpha}^{2}+k_{\alpha}^{2}\right) ; i, j, k=x, y, z ; \text { cyclic. }
$$

From [3a] we get for a linear molecule:

$$
\begin{gathered}
\sigma_{z z}^{d}=\sigma_{\|}^{d}=\sigma_{\mathrm{av}}^{d} \text { (free atom), } \\
\sigma_{x x}^{d}=\sigma_{y y}^{d}=\sigma_{\perp}^{d}=\sigma_{\mathrm{av}}^{d} \text { (free atom) }+\frac{e^{2}}{2 m c^{2}} \sum_{\alpha}^{\prime} \frac{N_{\alpha}}{r_{\alpha}} .
\end{gathered}
$$

Combining Eqs. $[2,4$, and 5] we get for the anisotropic chemical shift of a linear molecule

$$
\Delta \sigma=\sigma_{\|}-\sigma_{\perp}=-\frac{|e|}{2 m c} \frac{\Theta}{\mu_{0} g_{K}} C_{K} .
$$

Equation [6] shows that for linear molecules the anisotropy of the chemical shift is approximately proportional to the product of the moment of inertia and the spinrotation constant.

An expression for the relaxation rate of a nuclear spin due to spin-rotation interaction has been derived by Hubbard (8). For a linear molecule, we get (see also Green

${ }^{1} C_{x}$ is a component of the spin-rotation tensor defined by $\mathscr{H}_{\mathrm{an}}=\hbar \vec{I} \cdot C_{\mathrm{E}} \cdot \vec{J}$. Thus spinrotation constants obtained from molecular beam data have to be multiplied by $2 \pi$ to be used in our notation. 
and Powles (9))

$$
\frac{1}{T_{1}^{\mathrm{SR}}}=\frac{4}{3} \Theta \frac{k T}{\hbar^{2}} C_{\mathrm{K}}^{2} \tau_{\mathrm{SR}},
$$

where $\tau_{\mathrm{SR}}$ is the correlation time for angular velocity. Huntress $(10)$ has derived equations for the relaxation rate due to anisotropic chemical shift for a liquid in the diffusion limit. For a linear molecule we have

$$
\frac{1}{T_{1}^{\mathrm{CS}}}=\frac{1}{45} \frac{\omega^{2} \Theta}{k T}(\Delta \sigma)^{2} \frac{1}{\tau_{\mathrm{SR}}} .
$$

In the diffusion limit, the correlation times for angular velocity $\tau_{\mathrm{SR}}$ and angular reorientation $\tau_{c}$ are related by $(10,8)$

$$
\left(\tau_{c}\right)_{i}\left(\tau_{\mathrm{SR}}\right)_{i}=\frac{\Theta_{i}}{6 k T},
$$

where the index $i$ refers to the $i$-th axis. Therefore, Eq. [8] is identical with the expression usually given for $1 / T_{1}^{\mathrm{CS}}$ using $\tau_{c}(1,2)$

$$
\frac{1}{T_{1}^{\mathrm{CS}}}=\frac{2}{15} \omega^{2}(\Delta \sigma)^{2} \tau_{c}
$$

It is apparent from Eqs. $[7,8]$ that $\tau_{\mathrm{SR}}$ can be eliminated by multiplication of the two relaxation rates (see also Ref. (4)):

$$
\frac{1}{T_{1}^{\mathrm{SR}}} \cdot \frac{1}{T_{1}^{\mathrm{CS}}}=\frac{4}{135} \frac{\omega^{2} \Theta^{2}}{\hbar^{2}} C_{R}^{2}(\Delta \sigma)^{2} .
$$

If $1 / T_{1}^{\mathrm{SR}}$ and $1 / T_{1}^{\mathrm{CS}}$ can be determined experimentally, Eqs. $[6,10]$ can be used to obtain the absolute values of both $C_{K}$ and $\Delta \sigma$ from relaxation studies. It should be noted that the only molecular parameter besides $C_{K}$ and $\Delta \sigma$ appearing in Eqs. [6, 10] is the moment of inertia which is well defined for nonassociated liquids.

The close relationship of spin-rotation interaction and anisotropic chemical shift can also be used to calculate the spin-rotation constant for a given linear molecule from a known spin-rotation constant for the same nucleus in a different linear molecule if the relative chemical shift is known. Thus combining Eqs. [2] and [3] and noting that $\sigma_{\|}^{p}=0$ (see above), we get (7)

$$
\sigma_{\mathrm{av}}=\sigma_{\mathrm{av}}^{d}(\text { free atom })+\frac{|e|}{3 m c} \cdot \frac{\Theta}{\mu_{0} g_{\mathrm{K}}} C_{K} .
$$

Equation [11] is used below to calculate the ${ }^{13} \mathrm{C}$ spin-rotation constants for $\mathrm{CS}_{2}$ and $\mathrm{CO}_{2}$ based on the known value for ${ }^{13} \mathrm{CO}$.

\section{EXPERIMENTAL}

The $\mathrm{CS}_{2}$ sample, isotopically enriched $(60 \%)$ and oxygen-free, was obtained from Prochem Ltd., Carolyn House, Croydon, England. A Bruker pulse spectrometer was used together with a Siemens superconducting magnet (max. field $80 \mathrm{kG}$ ). The NMR probes, operating at 14,30 , and $62 \mathrm{MHz}$ were advanced versions of the single coil probe described earlier (11). All data were directly taken from the oscilloscope. 
Details of the method used to measure $T_{1}$ have also been described in Ref. (11). The sample temperature was controlled by a regulated nitrogen stream and determined by a thermo-couple ncar the sample; it was stable within $1^{\circ} \mathrm{C}$ during the time necessary to determine $T_{1}$ (typically ca. $30 \mathrm{~min}$ ).

\section{RESULTS AND DISCUSSION}

In Fig. 1 the experimental spin-lattice relaxation rates $1 / T_{1}$ obtained at 14,30 , and $62 \mathrm{MHz}$ are plotted vs. the temperature. The data were taken over essentially all of the liquid range at atmospheric pressure. So the temperatures are well below the critical temperature. It is apparent from Fig. 1 that relaxation through anisotropic

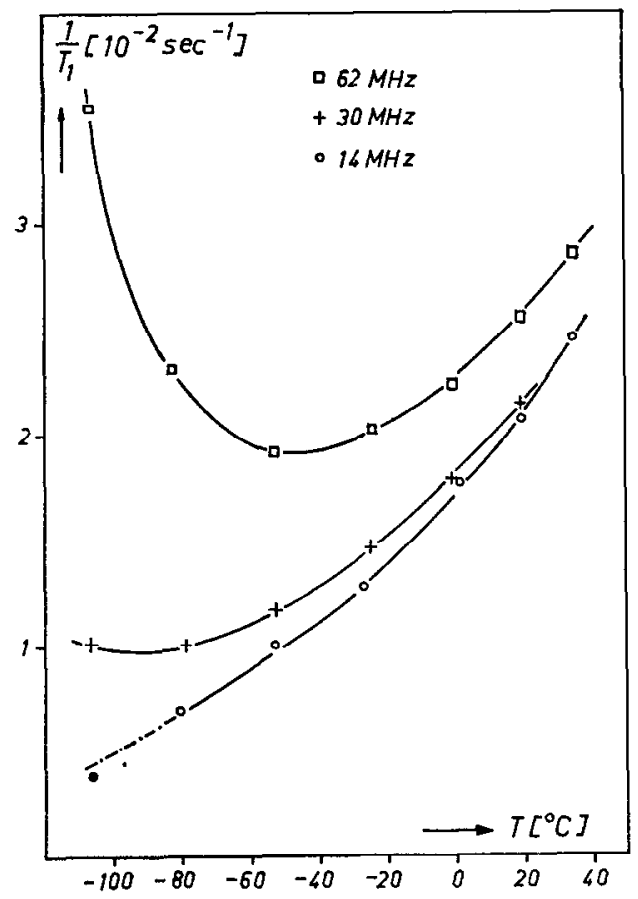

Fig. 1. Experimental relaxation rates in ${ }^{13} \mathrm{CS}_{2}$ at $14.0369,30.1026$, and $61.9164 \mathrm{MHz}$.

chemical shift is dominant for $62 \mathrm{MHz}$ at low temperatures. In order to separate the relaxation rate due to anisotropic chemical shift from the magnetic field independent part, in Fig. 2 the relaxation rates for the different temperatures vs. the square of the resonance frequency are plotted. Straight lines are obtained and therefore $1 / T_{1}^{\mathrm{CS}}$ can be determined accurately for $62 \mathrm{MHz}$.

The field-independent part of the relaxation rate decreases with decreasing temperature, suggesting that it is dominated by the spin-rotation interaction. The dipolar contribution to $1 / T_{1}$ can be estimated (2) to give for the intramolecular interaction $\left(1 / T_{1}^{d}\right)_{\text {intra }}<3 \cdot 10^{-6} \mathrm{sec}^{-1}$ and for the intermolecular interaction $\left(1 / T_{1}\right)_{\text {inter }}<$ $2 \cdot 10^{-5} \mathrm{sec}^{-1}$, which can be neglected compared with the measured relaxation rates 
(see Fig. 1). This was also confirmed experimentally by studying $T_{1}$ of a $\mathrm{CS}_{2}$ sample with ${ }^{13} \mathrm{C}$ in natural abundance at $62 \mathrm{MHz}$. No deviations from the isotopically enriched sample were found.

Thus we are left with only two relaxation mechanisms, and the analysis outlined above can be employed. Figure 3 shows the relaxation rates due to anisotropic chemical shift at $62 \mathrm{MHz}$ and due to spin-rotation interaction, together with the product of the two rates, which is independent of the temperature within experimental

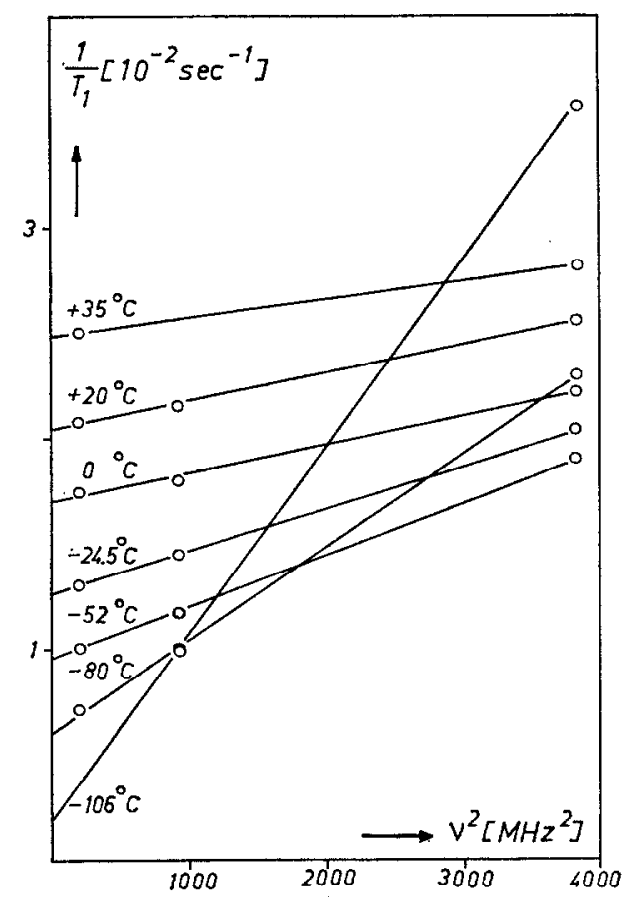

Fig. 2. Frequency dependence of experimental relaxation rates in ${ }^{13} \mathrm{CS}_{2}$.

error. This result confirms Eq. [10] experimentally, particularly since the individual relaxation rates span about the same range in the temperature interval studied.

In the derivation of Eq. [10] the relation between the correlation times for angular velocity and angular reorientation, Eq. [9] has been used. This relationship has recently been shown (12) to be valid for the quasi-spherical molecule $\mathrm{ClO}_{3} \mathrm{~F}$, where both $\tau_{c}$ and $\tau_{\mathrm{SR}}$ were determined experimentally, at temperatures significantly below the critical temperature. On the other hand, Deverell (13) has noted deviations from Eq. [9]. The values for $\tau_{c}$ he used, however, were calculated from macroscopic diffusion constants using estimated molecular radii. The possibility that the error in $\tau_{c}$ can account for at least part of the discrepancy is mentioned already in Ref. (13). This demonstrates that calculating $\tau_{c}$ using the Stokes-Einstein equation is not sufficient in order to get meaningful values for $\tau_{\mathrm{SR}}$ using Eq. [9] and thus reliable values for the spin-rotation constants from relaxation rates. 


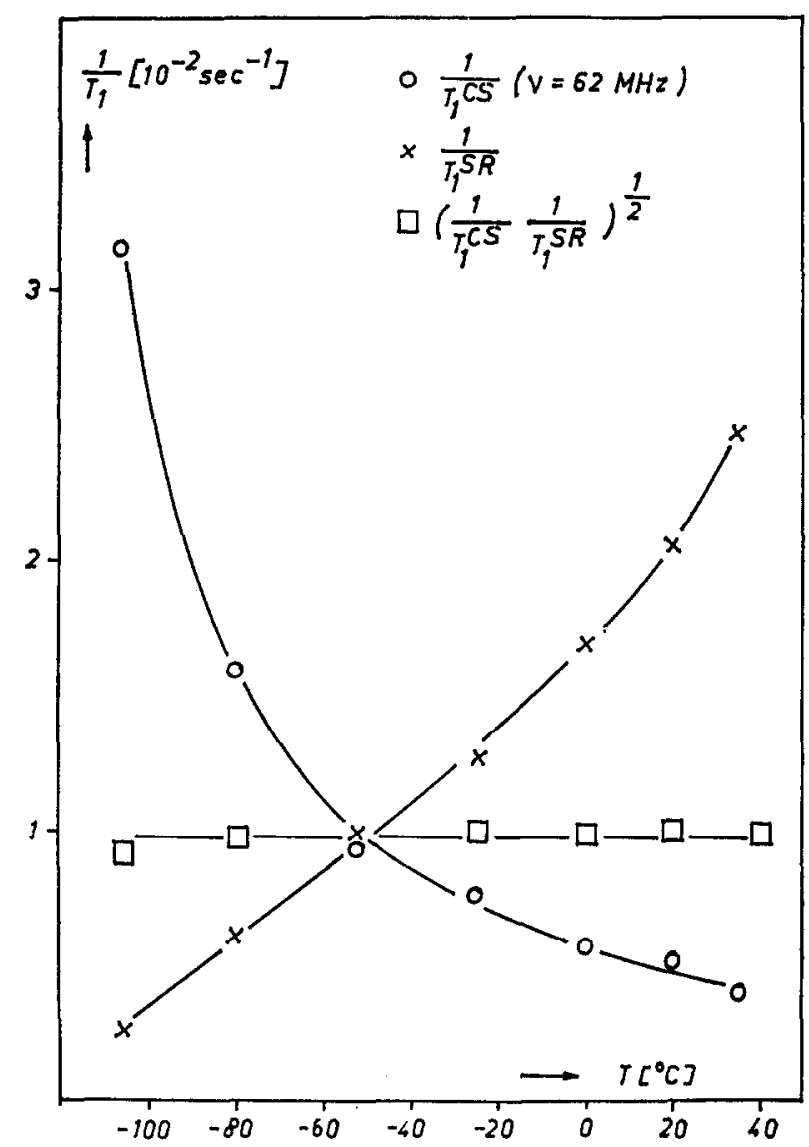

FIg. 3. Relaxation rates due to anisotropic chemical shift at $62 \mathrm{MHz}$ and due to spin-rotation interaction in ${ }^{13} \mathrm{CS}_{2}$.

Equation [9] is valid only if $\tau_{\mathrm{SR}} \ll \tau_{c}(8,10)$. An order of magnitude estimate may be obtained by calculating the correlation times using the macroscopic viscosity $(8)$ :

$$
\tau_{\mathrm{SR}}=\frac{1}{8} \Theta a^{-3} \pi \eta \quad \text { and } \quad \tau_{c}=\frac{4 \pi}{3} a^{3} \eta(k T)^{-1}
$$

As the ratio $\tau_{\mathrm{SR}} / \tau_{c}$ decreases with decreasing temperature we need only consider the highest temperature $\left(35^{\circ} \mathrm{C}\right)$. Using $a=1.5 \AA$, the internuclear distance C-S, we get $\tau_{\mathrm{SR}} \approx 3 \cdot 10^{-14} \mathrm{sec}$ and $\tau_{c} \approx 1.3 \cdot 10^{-12} \mathrm{sec}$ and thus $\tau_{\mathrm{SR}} \ll \tau_{c}$ is fulfilled. From the experimental data, we then get for ${ }^{13} \mathrm{C}$ in $\mathrm{CS}_{2}$, using Eqs. $[6,10]$

$$
\begin{aligned}
|C| & =13.8 \pm 1.4 \mathrm{kHz}, \\
|\Delta \sigma| & =438 \pm 44 \mathrm{ppm} .
\end{aligned}
$$

As can be seen from Fig. 3 , the error in the measured quantity $\left(1 / T_{1}^{\mathrm{SR}} \cdot 1 / T_{1}^{\mathrm{CS}}\right)^{1 / 2}$ is only $5 \%$. The error limits are given under the assumption that the diffusion model can be applied and it should be noted that the major error is introduced by using Eq. [6]. The relationship between $\Delta \sigma$ and $C$ expressed in Eq. [6] can be checked for 
the similar molecules $\mathrm{CO}$ and $\mathrm{COS}$, where both quantities are known from molecular beam data $(14,15)$. The agreement with experiment is $4 \%$ for $\mathrm{CO}$ and $10 \%$ for $\mathrm{COS}$ which is within the experimental error for $\Delta \sigma$.

The correlation times $\tau_{\mathrm{SR}}$ and $\tau_{c}$ calculated from the relaxation rates with these parameters are given in Table 1 . The values for $\tau_{c}$ agree quite well with correlation

TABLE 1

Correlation Times for Angular Velocity $\tau_{\mathrm{Sr}}$ and Angular Reorientation $\tau_{\mathrm{c}}$ in $\mathrm{CS}_{2}$

\begin{tabular}{lrr}
\hline$T\left({ }^{\circ} \mathrm{K}\right)$ & \multicolumn{1}{c}{$\tau_{\mathrm{gR}}(\mathrm{sec})$} & \multicolumn{1}{c}{$\tau_{\mathrm{c}}(\mathrm{sec})$} \\
\hline 308 & $10.0 \cdot 10^{-14}$ & $1.0 \cdot 10^{-12}$ \\
293 & $8.7 \cdot 10^{-14}$ & $1.4 \cdot 10^{-12}$ \\
273 & $7.6 \cdot 10^{-14}$ & $1.5 \cdot 10^{-12}$ \\
248.5 & $6.3 \cdot 10^{-14}$ & $2.1 \cdot 10^{-12}$ \\
221 & $5.4 \cdot 10^{-14}$ & $2.4 \cdot 10^{-12}$ \\
193 & $3.9 \cdot 10^{-14}$ & $4.1 \cdot 10^{-12}$ \\
167 & $1.8 \cdot 10^{-14}$ & $8.4 \cdot 10^{-12}$ \\
\hline
\end{tabular}

times estimated from light scattering data (16). It is seen that the diffusion model should be a good description at the lowest temperatures. For the highest temperature studied, on the other hand, the difference in the correlation times is only one order of magnitude. The experimental fact that $1 / T_{1}^{\mathrm{SR}} \cdot 1 / T_{1}^{\mathrm{CS}}$ is constant over the entire temperature range (Fig. 3) indicates, however, that even for the highest temperatures the deviations from the diffusion limit are within experimental error.

Spin-rotation constants are extremely difficult to compute from first principles as such a calculation depends on the knowledge of all the excited energy levels of the molecule. By use of Eq. [11], however, semiempirical values for the spin-rotation constant can be calculated with which our data may be compared. For linear molecules the anisotropy of the chemical shift can also be calculated from a known value of $\Delta \sigma$ for a different linear molecule and the relative isotropic chemical shift, using the assumption that $\sigma_{\|}$is constant for linear molecules as outlined above.

A molecule suitable for comparison is ${ }^{13} \mathrm{CO}$ : The spin-rotation constant of ${ }^{13} \mathrm{C}$ is known from molecular beam data with high accuracy (14), the anisotropy of the chemical shift is also given in Ref. (14), and the relative isotropic chemical shifts of ${ }^{13} \mathrm{CO},{ }^{13} \mathrm{CO}_{2}$, and ${ }^{13} \mathrm{CS}_{2}$ have been determined (17). In Table 2 values calculated in this way for $C$ and $\Delta \sigma$ are given for $\mathrm{CO}_{2}$ and $\mathrm{CS}_{2}$ together with the experimental data for $\operatorname{COS}(15)$. The experimental and calculated absolute values of $C$ and $\Delta \sigma$ for $\mathrm{CS}_{2}$ agree within experimental error. Furthermore, the comparison of the calculated values for $\mathrm{CO}_{2}$ and $\mathrm{CS}_{2}$ with the parameters measured for $\mathrm{COS}$ shows that the signs are also correct as obtained from the calculation. We, therefore, conclude that in $\mathrm{CS}_{2}$ the ${ }^{13} \mathrm{C}$ spin-rotation constant is negative and the anisotropy of the chemical shift is positive, as shown in Table 2 . The close agreement of calculated and observed 
TABLE 2

Calculated and Observed Values for Spin-rotation Constants and ANisotropic Chemical Shifts IN ${ }^{19} \mathrm{CO}_{2},{ }^{13} \mathrm{COS}$, and ${ }^{13} \mathrm{CS}_{2}$

\begin{tabular}{ccccc}
\hline & \multicolumn{2}{c}{$C(\mathrm{kHz})$} & \multicolumn{2}{c}{$\Delta \sigma \mathrm{ppm}$} \\
\cline { 2 - 5 } & exptl & calcd & exptl & calcd \\
\hline $\mathrm{CO}_{2}{ }^{a}$ & & -33.9 & & 315 \\
$\mathrm{COS}^{b}$ & $-19.5 \pm 1.3$ & & $372 \pm 42$ & \\
$\mathrm{CS}_{2}{ }^{a}$ & $-13.8 \pm 1.4$ & -12.6 & $438 \pm 44$ & 428 \\
\hline
\end{tabular}

${ }^{a}$ This work. ${ }^{b}$ See Ref. (15).

values demonstrates that our method of eliminating the correlation times allows the determination of spin-rotation constants and shift anisotropies from relaxation studies. This is particularly important for symmetric molecules like $\mathrm{CS}_{2}$, which are unsuitable for microwave or electric molecular beam resonance experiments.

Note added in proof: Recently $\Delta \sigma$ has been determind (18) in solid ${ }^{13} \mathrm{CS}_{2}$. The value obtained, $\Delta \sigma=425 \pm 15 \mathrm{ppm}$, compares remarkably well with our result.

\section{REFERENCES}

1. H. M. McConnell AND C. H. Holm, J. Chem. Phys. 25, 1289 (1956).

2. A. Abragam, "The principles of Nuclear Magnetism," Oxford University Press, Oxford, 1961.

3. E. LippmaA, T. PehK, A. Olivson, AND A. Süors, "Proc. XVI. Coll. Ampère," Bucharest, 1970.

4. J. M. Deutch and J. S. Waugh, J. Chem. Phys. 43, 1914 (1965).

5. N. F. RAMSEY, Phys. Rev. 78, 699 (1950).

6. W. H. Flygare, J. Chem. Phys. 41, 793 (1964).

7. W. H. Flygare and J. Goodisman, J. Chem. Phys. 49, 3122 (1968).

8. P. S. Hubbard, Phys. Rev. 131, 1155 (1963).

9. D. K. Green and J. G. Powles, Proc. Phys. Soc. 85, 87 (1965).

10. W. T. Huntress, J. Chem. Phys. 48, 3524 (1968).

11. H. JẌCKLe, U. HAEBerLen, AND D. SChWEITZER, J. Magn. Resonance, to appear.

12. A. A. Maryott, T. C. Farrar, and M. S. Malmberg, J. Chem. Phys. 54, 64 (1971).

13. C. Deverell, Mol. Phys. 18, 319 (1970).

14. I. Ozier, K. M. Crapo and N. F. Ramsey, J. Chem. Phys. 49, 2314 (1968).

15. F. H. De Leeuw and A. Dymanus, Chem. Phys. Lett. 7, 288 (1970).

16. S. L. Shapiro and H. P. Broida, Phys. Rev. 154, 129 (1967).

17. R. Ettinger, P. Blume, A. Patterson, JR., And P. C. Lauterdur, J. Chem. Phys. 33, 1597 (1960).

18. A. Pines, W. K. Rhim, and J. S. Waugh, to be published. 